This article may be downloaded for personal use only. Any other use requires prior permission of the author and AIP Publishing.

This article appeared in Surface Science Spectra 27, 024014 (2020) and may be found at https://avs.scitation.org/doi/10.1116/6.0000472. 


\section{Niobium ethoxide analyzed by XPS}

Sergio A. Rincón-Ortiz ${ }^{1}$, Jhonatan Rodriguez-Pereira ${ }^{1,2}$ and Rogelio Ospina ${ }^{1, a)}$

${ }^{1}$ Centro de Investigación Científica y Tecnológica en Materiales y Nanociencias (CMN), Universidad Industrial de Santander, Piedecuesta, Santander, P.C. 681011, Colombia.

${ }^{2}$ Center of Materials and Nanotechnologies, Faculty of Chemical Technology, University of Pardubice, Nam. Cs. Legii 565, 53002 Pardubice, Czech Republic.

(Received day Month year; accepted day Month year; published day Month year)

Niobium (V) ethoxide was characterized by x-ray photoelectron spectroscopy (XPS). The specimen is a powder purchased from Sigma Aldrich. Sample was fixed to a stainless-steel sample holder with copper double-sided adhesive tape. Survey spectra, Nb 3d, O 1s, C 1s, Nb $3 p, \mathrm{Nb} 4 \mathrm{p}, \mathrm{O} 2 \mathrm{~s}$ core levels and valence band spectra were acquired. Results showed how the elements in niobium ethoxide structure are bonded.

Keywords: Niobium ethoxide; XPS; precursor; niobium ethanolate
Accession\#: 01676

Technique: XPS

Host Material: Niobium ethoxide

Instrument: SPECS

PHOIBOS 150

Major Elements in Spectra: $\mathrm{Nb}, \mathrm{O}, \mathrm{C}$

Minor Elements in Spectra: None

Published Spectra: 6

Spectra in Electronic Record: 6

Spectral Category: comparison

\section{INTRODUCTION}

Niobium (V) ethoxide (Fig. 1) is a colorless to yellow organometallic compound, easily hydrolyzable and soluble in some organic solvents such as ethanol, it has a relative density of $1.268 \mathrm{~g} / \mathrm{cm}^{3}$ at $25^{\circ} \mathrm{C}$. It is widely used as a precursor for different processes, such as the generation of niobium oxide films by CVD (Refs 1 - 3), for applications in devices in the electronic and optical industry. Another important method that uses niobium ethoxide as a precursor to generate $\mathrm{NbO}$ or $\mathrm{LiNbO}_{3}$ films is the sol-gel Refs. 3 - 5). These niobium oxides have a very high dielectric constant in thin films, being used by this property for memories (DRAMs) (Ref. 6). Furthermore, it is also used to produce $\mathrm{Nb}_{2} \mathrm{O}_{5}$, which is widely used for photocatalytic processes (Ref. 7).

C

Fig. 1. Structure of niobium ethoxide

XPS survey spectrum indicates that there are no intrusive elements in the sample, since the presence of just carbon, oxygen and niobium is evidenced.

$\mathrm{Nb} 3 \mathrm{~d}$ high resolution spectrum was fitted with four contributions corresponding to two different chemical species. The peaks centered at 207.2 and $209.9 \mathrm{eV}$ were assigned to niobium bonded with oxygen, O-Nb-O, and signals at 208.4 and $211.1 \mathrm{eV}$ were associated with niobium bonded with oxygen related with carbon, Nb-O-C. High resolution spectrum of O 1 s shows three chemical environments. O-Nb-O at $530.5 \mathrm{eV}$, oxygen bonded with bismuth. C-O at $532.2 \mathrm{eV}$, oxygen single bonded with carbon. And, C-O-Nb at $533.7 \mathrm{eV}$, oxygen bonded with carbon and niobium. C 1s spectrum displays three chemical species. First, carbon carbon bonding or adventitious carbon, $\mathbf{C}-(\mathrm{C}, \mathrm{H})$ at 284.8 $\mathrm{eV}$ used as a reference (Ref. 8). Second, carbon single bounded with oxygen, C-O at $286.5 \mathrm{eV}$. And, carbon sinlge bonded with oxygen related with niobium, $\mathrm{C}-\mathrm{O}-\mathrm{Nb}$ at $288.8 \mathrm{eV}$.

\section{SPECIMEN DESCRIPTION (ACCESSION \# 01676)}

Host Material: Niobium ethoxide

CAS Registry \#: 3236-82-6

Host Material Characteristics: homogeneous; solid; polycrystalline; unknown conductivity; metalorganic compound; Powder

Chemical Name: Niobium ethoxide

Source: Sigma Aldrich

Host Composition: Niobium (V) ethoxide (99.95\%)

Form: Powder

Structure: $\mathrm{Nb}\left(\mathrm{OCH}_{2} \mathrm{CH}_{3}\right)_{5}$

History \& Significance: Niobium (V) ethoxide powder was ground and fixed to a sample holder with copper $3 \mathrm{M}^{\mathrm{TM}}$ doublesided adhesive tape. The sample was exposed to the environment for about 2 minutes, time that was spent to prepare the sample and then introduce it to the platform.

As Received Condition: As powder

Analyzed Region: same as host material

Ex Situ Preparation/Mounting: As received.

In Situ Preparation: None 
Charge Control: Electron flood gun (SPECS FG-500) operated at $70 \mu \mathrm{A}$ and $4 \mathrm{eV}$

Temp. During Analysis: $300 \mathrm{~K}$

Pressure During Analysis: $<1 \times 10^{-7} \mathrm{~Pa}$

Pre-analysis Beam Exposure: $60 \mathrm{~s}$

\section{INSTRUMENT DESCRIPTION}

Manufacturer and Model: SPECS PHOIBOS 150 - 2D-DLD - SPECS Surface Nano Analysis GmbH

Analyzer Type: spherical sector

Detector: other

Number of Detector Elements: 25

INSTRUMENT PARAMETERS COMMON TO ALL SPECTRA

-Spectrometer

Analyzer Mode: constant pass energy

Throughput $\left(T=E^{N}\right): N=0$

Excitation Source Window: Mylar window, allows high Xray transmission: $88 \%$ for $\mathrm{Al} \mathrm{K} \alpha$.

Excitation Source: Al Ka monochromatic

Source Energy: $1486.6 \mathrm{eV}$

Source Strength: $200 \mathrm{~W}$

Source Beam Size: $2000 \mu \mathrm{m}$ x $2000 \mu \mathrm{m}$

Signal Mode: multichannel direct

-Geometry

Incident Angle: $55^{\circ}$

Source-to-Analyzer Angle: $55^{\circ}$

Emission Angle: $0^{\circ}$

Specimen Azimuthal Angle: Not applicable

Acceptance Angle from Analyzer Axis: $16^{\circ}$

Analyzer Angular Acceptance Width: $16^{\circ}$ x $16^{\circ}$

-lon Gun

Manufacturer and Model: SPECS IQE 12/38

Energy: $5000 \mathrm{eV}$

Current: $70 \mathrm{~mA}$

Current Measurement Method: biased stage

Sputtering Species: $\mathrm{Ar}^{+}$

Spot Size (unrastered): $3000 \mu \mathrm{m}$ x $3000 \mu \mathrm{m}$

Raster Size: Not applicable $\mu \mathrm{m} \times \mu \mathrm{m}$

Incident Angle: $54^{\circ}$

Polar Angle: $55^{\circ}$

Azimuthal Angle: $45^{\circ}$

Comment: The specimen was analyzed as loaded. The ion gun was used only for cleaning the Ag reference foil.
Energy Scale Correction: Binding energy of the adventitious carbon, C-(C,H) at $284.8 \mathrm{eV}$ (Ref. 8) was used as reference to adjust the binding energy scale of the spectra.

Recommended Energy Scale Shift: $2.76 \mathrm{eV}$

Peak Shape and Background Method: Peak position and width were determined from fitting the spectra using a mixed Gaussian- Lorentzian, GL (30) function after subtraction of a Shirley background using the CasaXPS Software.

Quantitation Method: Peak areas were obtained from fitting the spectra and relative sensitivity factors from the atomic photoionization cross section of each core level provided by SPECS Prodigy library.

\section{ACKNOWLEDGMENTS}

Authors thank to Laboratorio Central en Ciencia de Superficies (SurfLab-UIS) from Universidad Industrial de Santander for providing their facilities to perform the XPS measurements and to project 2321 of Vicerrectoría de Investigación y Extensión (VIE-UIS) from Universidad Industrial de Santander.

\section{REFERENCES}

1. Y. Cai, S. Yang, S. Jing, H. Yang, G. Hou and J. Xia, J. Cent. South Univ. Technol., 18, 73 (2011).

2. J.P. Masse, H. Szymanowski, O. Zabeida, A. Amassian, J.E. Klemberg-Sapieha and L. Martinu., Thin Solid Films, 515, 1674 (2006).

3. A. Tanaka, K. Miyashita, T. Tashiro, M. Kimura and T. Sukegawa, J. Cryst. Growth, 148, 324 (1995).

4. B.D. Wood, V. Moncanu and B.D. Gates, Adv. Mater., 20, 4552 (2008).

5. Y. Akiyama, K. Shitanaka, H. Murakami, Y.S. Shin, M. Yoshida and N. Imaishi, Thin Solid Films, 515, 4975 (2007)

6. A. Pignolet, G. Mohan Rao and S.B. Krupanidhi, Thin Solid Films, 261, 18 (1995).

7. C. Jaramillo-Páez, F.J. Sánchez-Fernández, J.A. Navío and M.C. Hidalgo, J. Photochem. Photobiol., A, 359, 40 (2018).

8. P. G. Rouxhet and M. J. Genet, Surf. Interface Anal. 43, 1453 (2011). 


\begin{tabular}{|c|c|c|c|c|c|c|c|}
\hline \multicolumn{8}{|c|}{ SPECTRAL FEATURES TABLE } \\
\hline $\begin{array}{c}\text { Spectrum } \\
\text { ID \# }\end{array}$ & $\begin{array}{c}\text { Element/ } \\
\text { Transition }\end{array}$ & $\begin{array}{c}\text { Peak } \\
\text { Energy } \\
(\mathrm{eV})\end{array}$ & $\begin{array}{c}\text { Peak } \\
\text { Width } \\
\text { FWHM } \\
\text { (eV) }\end{array}$ & $\begin{array}{l}\text { Peak Area } \\
(\mathrm{eV} \times \mathrm{cts} / \mathrm{s})\end{array}$ & $\begin{array}{l}\text { Sensitivity } \\
\text { Factor }\end{array}$ & $\begin{array}{c}\text { Concentration } \\
\text { (at. \%) }\end{array}$ & Peak Assignment \\
\hline 01676-02 & $\mathrm{Nb} 3 \mathrm{~d}$ & $\ldots$ & $\bar{\ldots}$ & $1.35 \times 10^{4}$ & 8.21 & 17.72 & \\
\hline 01676-02 & $\mathrm{Nb} 3 d_{5 / 2}$ & 207.2 & 1.37 & $\ldots$ & $\ldots$ & $\ldots$ & O-Nb-O \\
\hline 01676-02 & $\mathrm{Nb} 3 \mathrm{~d}_{3 / 2}$ & 209.9 & 1.37 & $\ldots$ & $\ldots$ & $\ldots$ & $\mathrm{O}-\mathrm{Nb}-\mathrm{O}$ \\
\hline 01676-02 & $\mathrm{Nb} 3 d_{5 / 2}$ & 208.4 & 1.78 & $\ldots$ & $\ldots$ & $\ldots$ & $\mathrm{Nb}-\mathrm{O}-\mathrm{C}$ \\
\hline 01676-02 & $\mathrm{Nb} 3 \mathrm{~d}_{3 / 2}$ & 211.1 & 1.78 & $\cdots$ & $\cdots$ & $\cdots$ & $\mathrm{Nb}-\mathrm{O}-\mathrm{C}$ \\
\hline 01676-03 & $01 s$ & $\ldots$ & $\ldots$ & $1.03 \times 10^{4}$ & 2.77 & 44.79 & \\
\hline 01676-03 & 0 1s & 530.5 & 1.62 & $\ldots$ & $\ldots$ & $\ldots$ & $\mathrm{O}-\mathrm{Nb}-\mathrm{O}$ \\
\hline 01676-03 & 0 1s & 532.2 & 1.62 & $\ldots$ & $\ldots$ & $\ldots$ & C-O \\
\hline 01676-03 & $01 \mathrm{~s}$ & 533.7 & 1.62 & $\ldots$ & $\ldots$ & $\ldots$ & $\mathrm{C}-\mathrm{O}-\mathrm{Nb}$ \\
\hline 01676-04 & C 1s & $\ldots$ & & $3.42 \times 10^{3}$ & 1.00 & 37.49 & \\
\hline 01676-04 & C 1s & 284.8 & 1.79 & $\ldots$ & $\ldots$ & $\ldots$ & $\mathbf{C}-(\mathrm{C}, \mathrm{H})$ \\
\hline 01676-04 & C $1 \mathrm{~s}$ & 286.5 & 1.79 & $\ldots$ & $\cdots$ & $\cdots$ & C-O \\
\hline 01676-04 & C 1s & 288.8 & 1.79 & $\ldots$ & $\ldots$ & $\ldots$ & $\mathrm{C}-\mathrm{O}-\mathrm{Nb}$ \\
\hline 01676-05 & $\mathrm{Nb} 3 p_{3 / 2}$ & 365.9 & 2.71 & $6.42 \times 10^{3}$ & $\ldots$ & $\ldots$ & $\ldots$ \\
\hline 01676-05 & $\mathrm{Nb} 3 \mathrm{p}_{1 / 2}$ & 381.1 & 2.83 & $4.35 \times 10^{3}$ & $\ldots$ & $\ldots$ & $\ldots$ \\
\hline 01676-06 & $\mathrm{Nb} 4 \mathrm{p}$ & 34.9 & 3.58 & $1.73 \times 10^{3}$ & $\ldots$ & $\ldots$ & $\ldots$ \\
\hline 01676-06 & $02 \mathrm{~s}$ & 22.7 & 2.52 & $5.74 \times 10^{2}$ & $\ldots$ & $\ldots$ & $\ldots$ \\
\hline $01676-06^{a}$ & VBM & 2.35 & $\ldots$ & $\ldots$ & $\ldots$ & $\ldots$ & $\ldots$ \\
\hline
\end{tabular}

\begin{tabular}{|c|c|c|c|c|c|c|c|}
\hline \multicolumn{8}{|c|}{ ANALYZER CALIBRATION TABLE } \\
\hline $\begin{array}{c}\text { Spectrum ID } \\
\# \\
\end{array}$ & $\begin{array}{l}\text { Element/ } \\
\text { Transition }\end{array}$ & $\begin{array}{c}\text { Peak Energy } \\
(\mathrm{eV})\end{array}$ & $\begin{array}{l}\text { Peak Width } \\
\text { FWHM (eV) }\end{array}$ & $\begin{array}{l}\text { Peak Area } \\
\text { (eV } \times \text { cts } / \mathrm{s}) \\
\end{array}$ & $\begin{array}{l}\text { Sensitivity } \\
\text { Factor }\end{array}$ & $\begin{array}{c}\text { Concentration } \\
\text { (at. \%) }\end{array}$ & $\begin{array}{c}\text { Peak } \\
\text { Assignment }\end{array}$ \\
\hline$\ldots$ & $\operatorname{Ag~3d_{5/2}}$ & 368.3 & 0.50 & $0.15 \times 10^{6}$ & $\ldots$ & $\ldots$ & $\ldots$ \\
\hline
\end{tabular}


GUIDE TO FIGURES

\begin{tabular}{|c|c|c|c|c|c|}
\hline $\begin{array}{l}\text { Spectrum } \\
\text { (Accession) \# }\end{array}$ & Spectral Region & Voltage Shift* & Multiplier & Baseline & Comment \# \\
\hline $01676-01$ & Survey & 0 & 1 & 0 & 1 \\
\hline 01676-02 & $\mathrm{Nb} 3 d$ & -2.76 & 1 & 0 & 1 \\
\hline $01676-03$ & $01 s$ & -2.76 & 1 & 0 & 1 \\
\hline 01676-04 & C 1s & -2.76 & 1 & 0 & 1 \\
\hline 01676-05 & $\mathrm{Nb} 3 p$ & -2.76 & 1 & 0 & 1 \\
\hline 01676-06 & $\mathrm{Nb} 4 \mathrm{p}, \mathrm{O} 2 \mathrm{~s}, \mathrm{VB}$ & -2.76 & 1 & 0 & 1 \\
\hline
\end{tabular}

*Voltage shift of the archived (as-measured) spectrum relative to the printed figure. The figure reflects the recommended energy scale correction due to a calibration correction, sample charging, flood gun, or other phenomenon.

1, Bismuth (III) acetate powder 


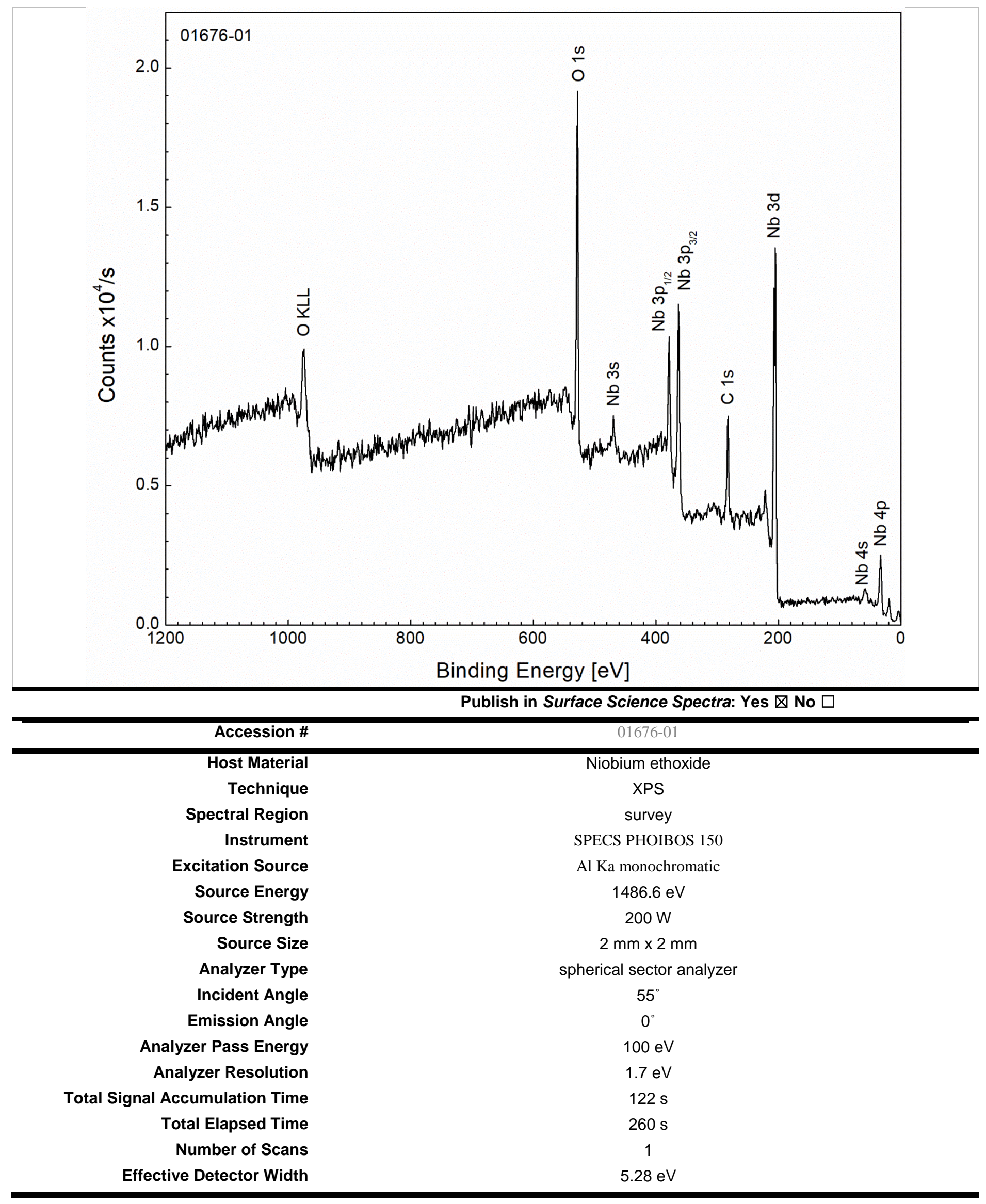




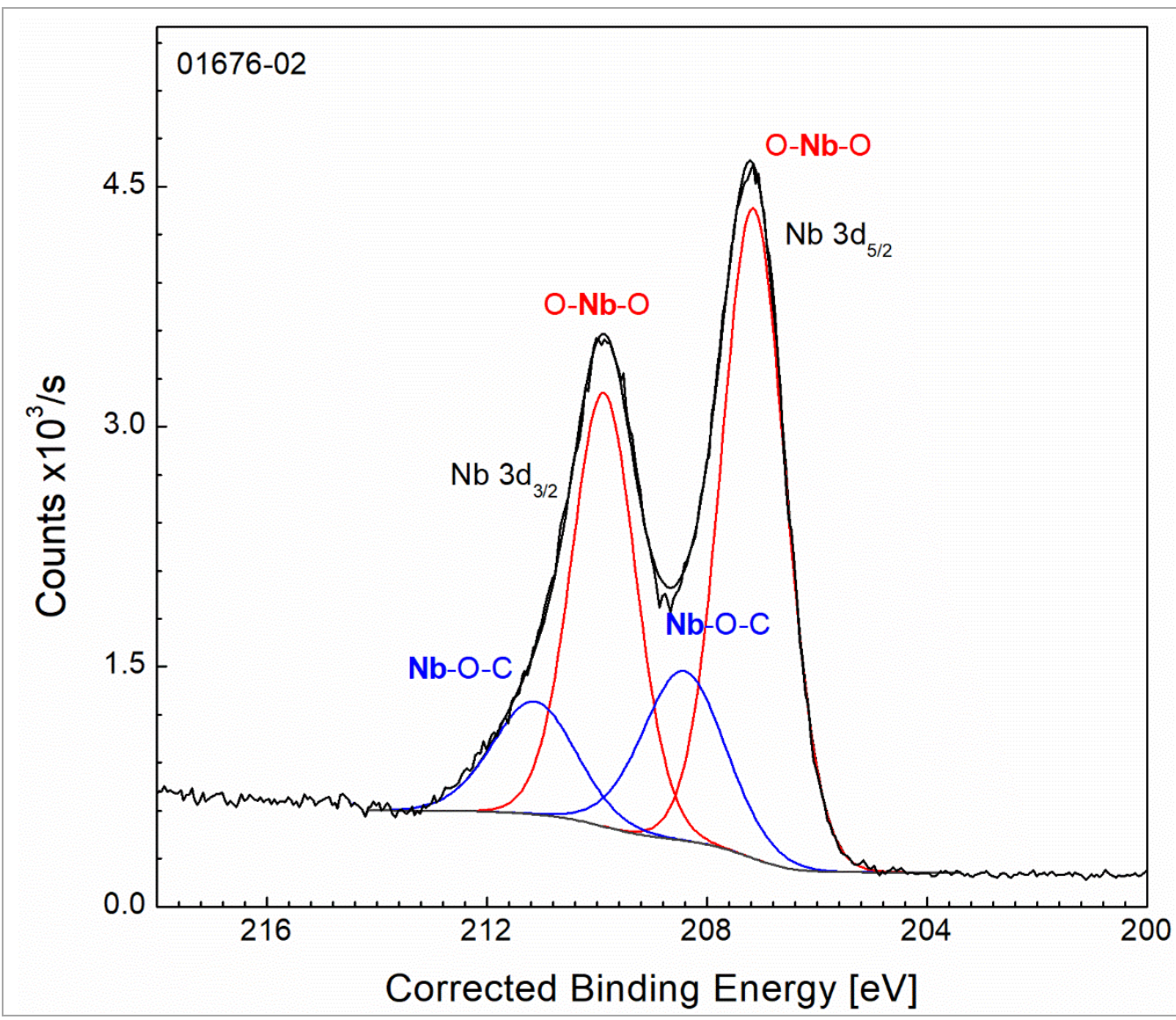

Publish in SSS: Yes $\otimes$ No $\square$

- Accession \#: 01676-02

- Host Material: Niobium ethoxide

- Technique: XPS

- Spectral Region: Nb 3d Instrument: SPECS PHOIBOS 150

Excitation Source: Al Ka monochromatic

Source Energy: $1486.6 \mathrm{eV}$

Source Strength: $200 \mathrm{~W}$

Source Size: $2 \mathrm{~mm} \times 2 \mathrm{~mm}$

Analyzer Type: spherical sector

Incident Angle: $55^{\circ}$

Emission Angle: 0

Analyzer Pass Energy $30 \mathrm{eV}$

Analyzer Resolution: $0.5 \mathrm{eV}$

Total Signal Accumulation Time: $801 \mathrm{~s}$

Total Elapsed Time: $1271 \mathrm{~s}$

Number of Scans: 8

Effective Detector Width: $2.64 \mathrm{eV}$

Publish in SSS: Yes $\triangle$ No $\square$

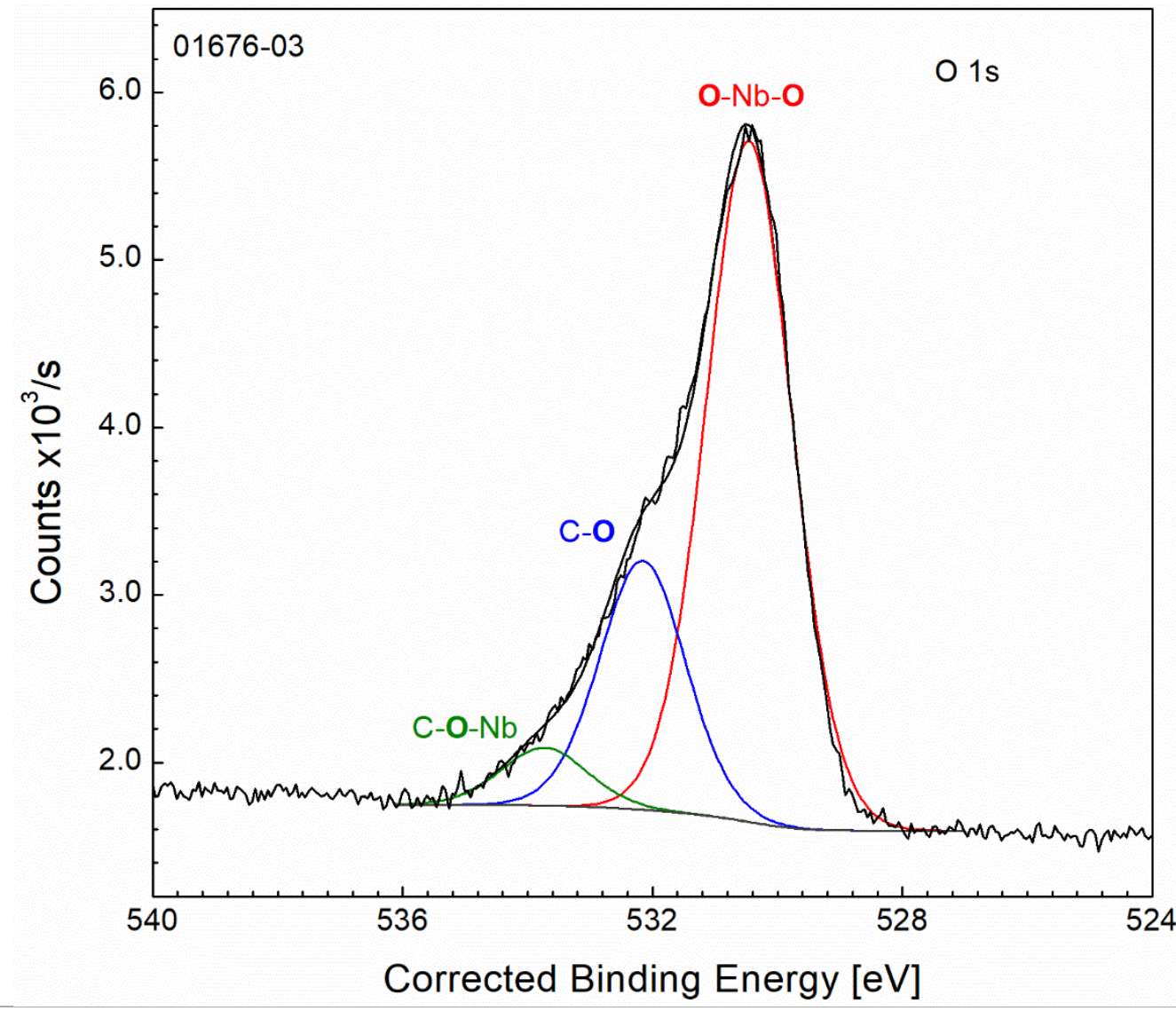

- Accession \#: 01676-03

- Host Material: Niobium ethoxide

- Technique: XPS

- Spectral Region: O 1s

Instrument: SPECS PHOIBOS 150

Excitation Source: Al Ka

monochromatic

Source Energy: $1486.6 \mathrm{eV}$

Source Strength: $200 \mathrm{~W}$

Source Size: $2 \mathrm{~mm} \times 2 \mathrm{~mm}$

Analyzer Type: spherical sector

Incident Angle: $55^{\circ}$

Emission Angle: $0{ }^{\circ}$

Analyzer Pass Energy 30 eV

Analyzer Resolution: $0.5 \mathrm{eV}$

Total Signal Accumulation Time: $421 \mathrm{~s}$

Total Elapsed Time: $668 \mathrm{~s}$

Number of Scans: 8

Effective Detector Width: 2.64 eV 


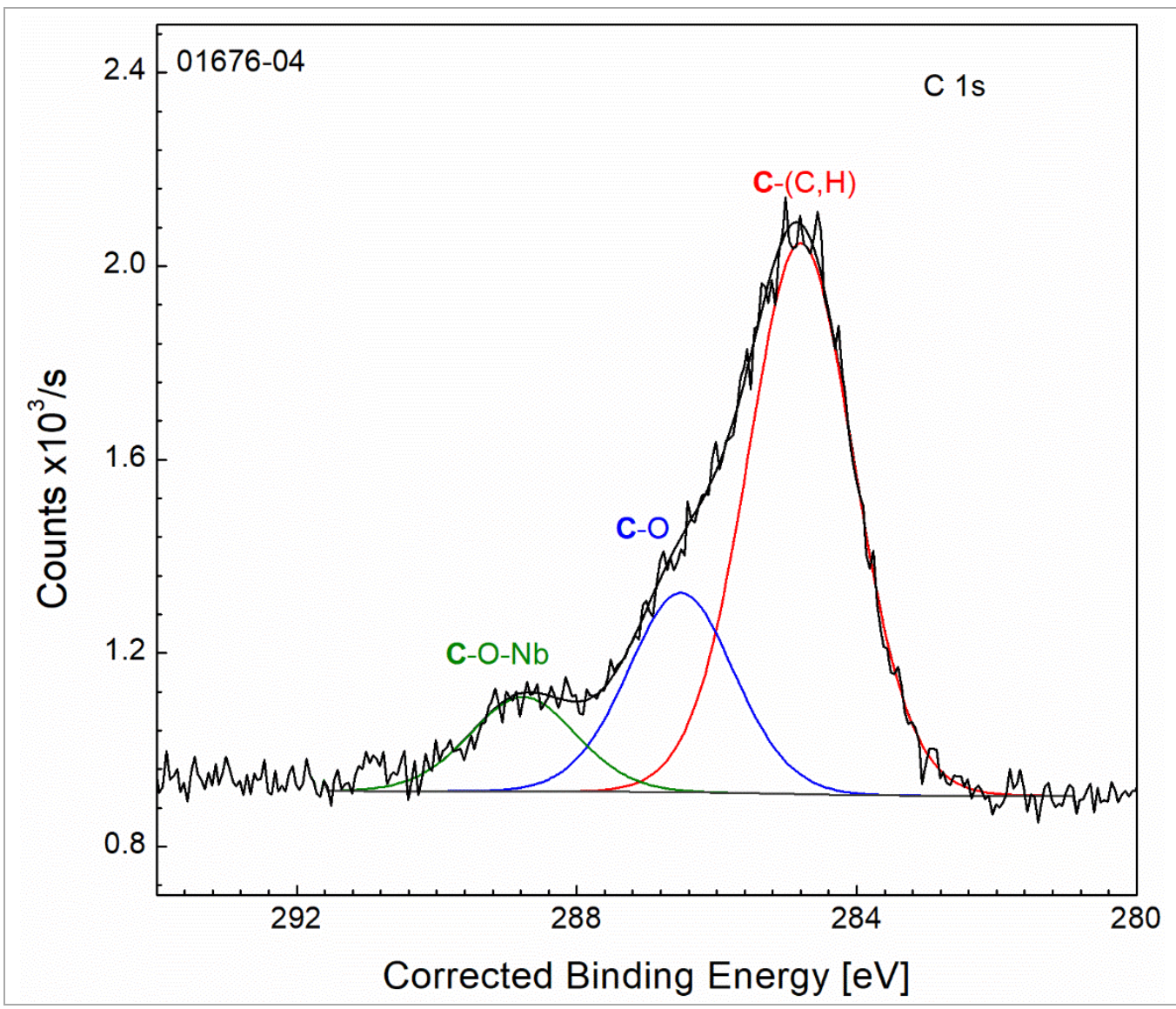

Publish in SSS: Yes $\otimes$ No $\square$

- Accession \#: 01676-04

- Host Material: Niobium ethoxide

- Technique: XPS

- Spectral Region: C 1s Instrument: SPECS PHOIBOS 150

Excitation Source: Al Ka monochromatic

Source Energy: $1486.6 \mathrm{eV}$

Source Strength: $200 \mathrm{~W}$

Source Size: $2 \mathrm{~mm} \times 2 \mathrm{~mm}$

Analyzer Type: spherical sector

Incident Angle: $55^{\circ}$

Emission Angle: $0^{\circ}$

Analyzer Pass Energy $30 \mathrm{eV}$

Analyzer Resolution: $0.5 \mathrm{eV}$

Total Signal Accumulation Time: $421 \mathrm{~s}$

Total Elapsed Time: $668 \mathrm{~s}$

Number of Scans: 8

Effective Detector Width: $2.64 \mathrm{eV}$

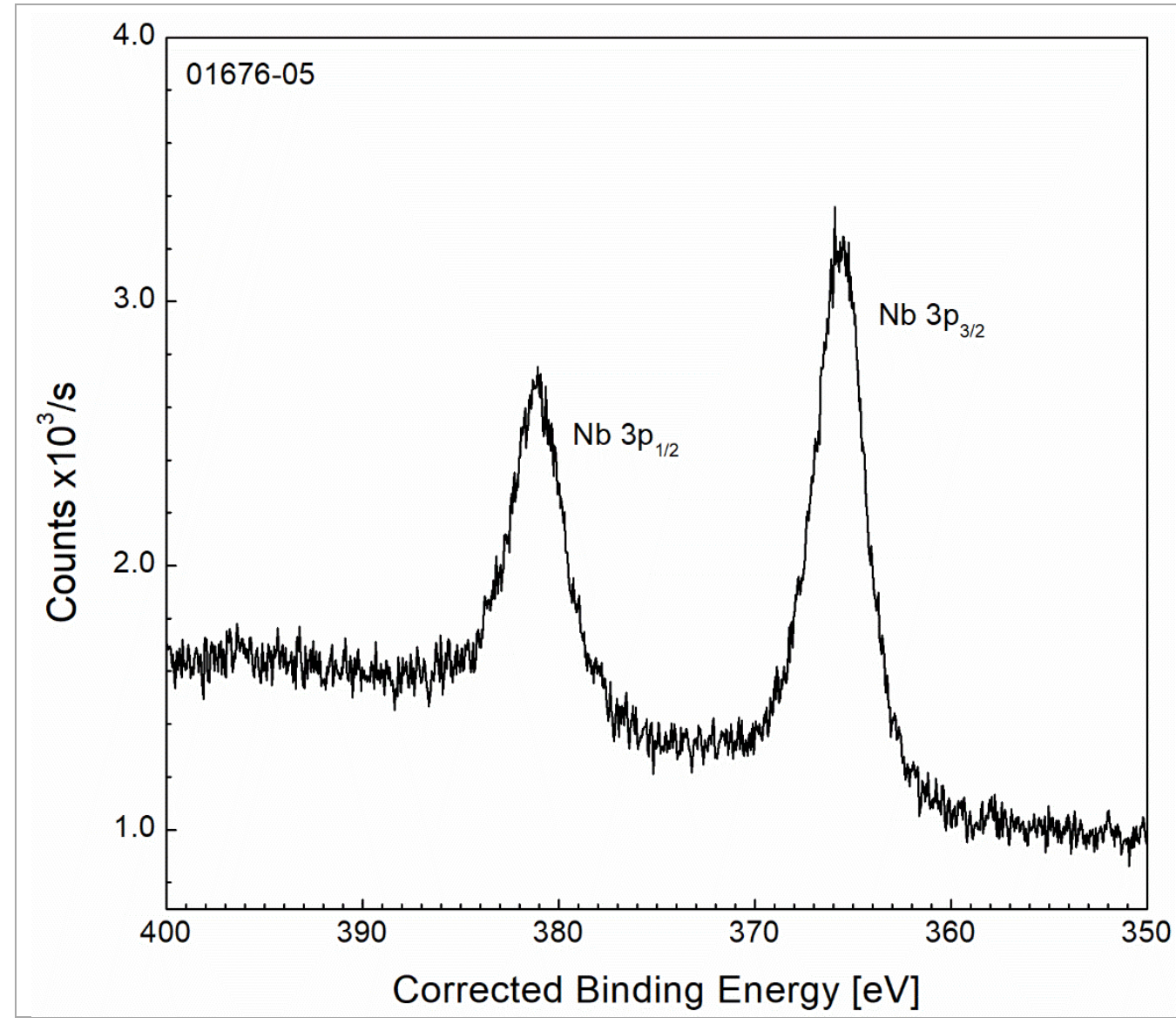

Publish in SSS: Yes $\triangle$ No $\square$

- Accession \#: 01676-05

- Host Material: Niobium ethoxide

- Technique: XPS

- Spectral Region: Nb 3p Instrument: SPECS PHOIBOS 150

Excitation Source: Al Ka monochromatic Source Energy: $1486.6 \mathrm{eV}$

Source Strength: $200 \mathrm{~W}$ Source Size: $2 \mathrm{~mm} \times 2 \mathrm{~mm}$ Analyzer Type: spherical sector Incident Angle: $55^{\circ}$

Emission Angle: 0 Analyzer Pass Energy $30 \mathrm{eV}$ Analyzer Resolution: $0.5 \mathrm{eV}$ Total Signal Accumulation Time: 1201 $\mathrm{s}$ Total Elapsed Time: 1906 s

Number of Scans: 4

Effective Detector Width: $2.64 \mathrm{eV}$ 


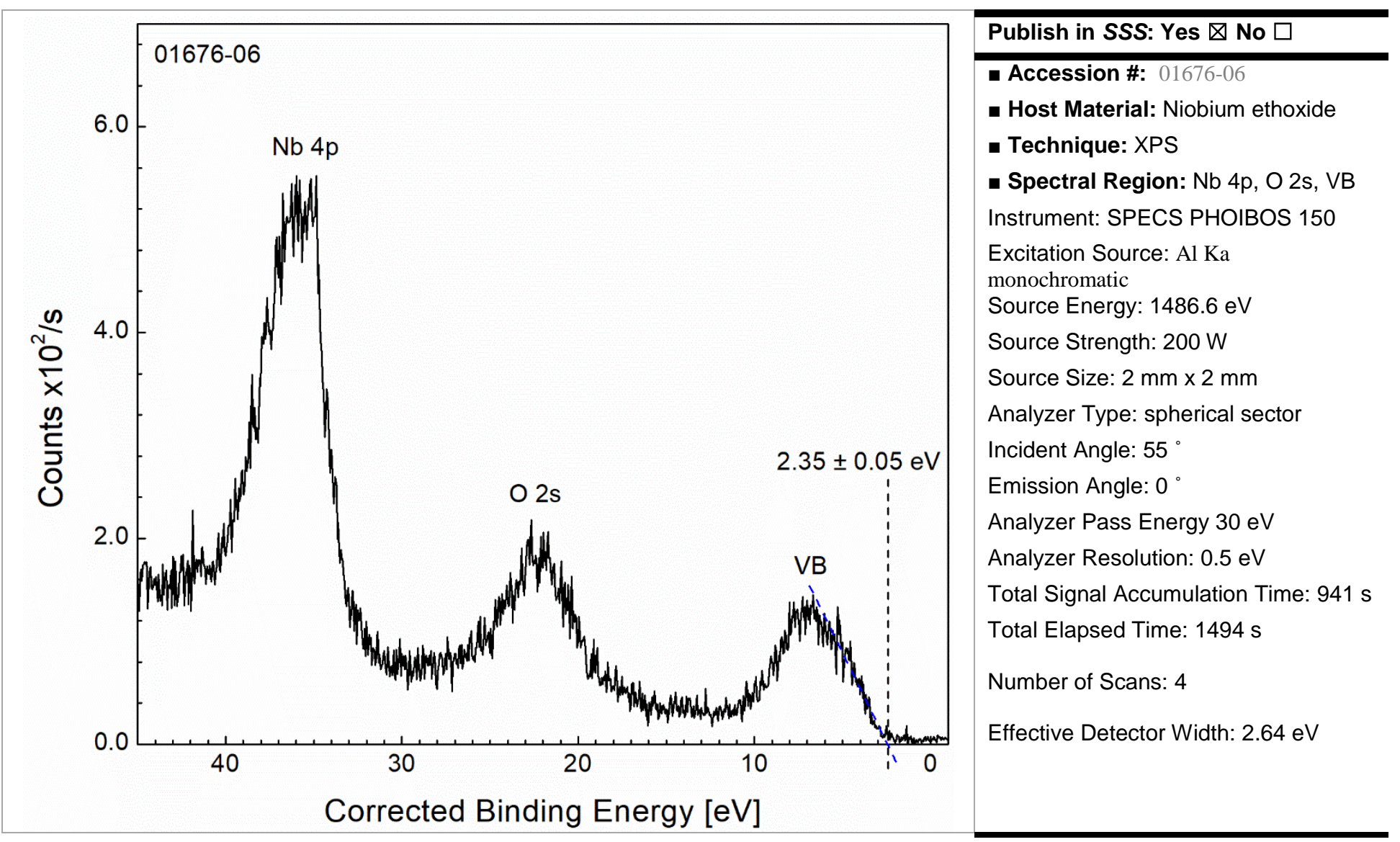

